\title{
Das Thema Flucht im Unterricht im digitalen Zeitalter - Welche Strategien nutzen Studierende, um „alternatives“ Unterrichtsmaterial zu evaluieren?
}

\begin{abstract}
Zusammenfassung
„Alternative" Unterrichtsmaterialien (als im Internet frei zugängliche, in der Regel nicht staatlich geprüfte Materialien unterschiedlichen Ursprungs) gewinnen im digitalen Zeitalter neben dem Schulbuch immer mehr an Bedeutung. Lehrkräfte greifen als Inspiration oder Ergänzung darauf zurück, vor allem auch um aktuelle Themen, wie zum Beispiel das Thema Flucht und Geflüchtete, im Unterricht aufzugreifen. Träger wie Nichtregierungsorganisationen (NROs) oder Stiftungen vermögen Themen zügiger pädagogisch aufzubereiten als beispielsweise Schulbuchverlage. Das große Angebot an Unterrichtsmaterialien und deren Prüfung stellt allerdings komplexe Anforderungen an Lehrkräfte hinsichtlich deren Auswahl und Evaluation. Die vorliegende Studie geht in diesem Kontext empirisch der Frage nach, wie angehende Lehrkräfte alternatives Material zum Thema Flucht evaluieren - also welche Strategien die Studierenden zur Auswahl und zur Bewertung des Materials heranziehen. Datengrundlage sind Gruppendiskussionen $(N=22)$, in denen Grundschullehramtsstudierende ausgewähltes ,alternatives“ Material diskutieren. In der rekonstruktiven Auswertung mit der Dokumentarischen Methode wurden zwei unterschiedliche Evaluationsstrategien herausgearbeitet, die im Hinblick auf Anforderungen in der Lehrkräftebildung diskutiert werden.
\end{abstract}

Schlüsselworte: Flucht, Geflüchtete, Grundschule, Unterrichtsmaterial, Sachunterricht, Grundschullehrkräfte

\footnotetext{
Abstract

"Alternative" educational material (as material of differing origin, which is freely accessible on the Internet and generally not state-approved) become increasingly important in the digital age, alongside the schoolbook. Teachers use it as an inspiration or complement, especially to address current topics, such as flight and refugees, in class. Organizations such as non-governmental organisations (NGOs) or foundations are able to prepare topics for educational aims more quickly than, for exam-
}

ple, school book publishers. However, the wide range of teaching materials and their assessment is demanding for teachers in terms of their choice and evaluation. This study empirically examines how future teachers evaluate an alternative material on flight - i.e. which strategies the students use to select and evaluate the material. The data is based on group discussions $(N=22)$ in which elementary school students discuss a selected 'alternative' material. In the reconstructive analysis with the documentary method two different evaluation strategies were identified, which are discussed with regard to requirements for teacher training.

Keywords: flight, refugees, primary school, teaching material, social and science studies, primary school teachers

\section{Das Angebot von „alternativen“ Unter- richtsmaterialien im digitalen Zeitalter}

Schnell mal ein illustrierendes Bild oder ein passendes Arbeitsblatt zum Unterrichtsthema recherchieren, die Fragen der Schülerinnen und Schüler über das Klassenpadlet beantworten oder die Idee für die nächste Unterrichtsstunde in einem Forum entdecken - für Lehrkräfte, die sich auf die Nutzungsmöglichkeiten des Internets einlassen, hat sich das Unterrichten verändert. Dazu gehört vornehmlich die Frage nach der Auswahl und der Evaluation von Unterrichtsmaterialien. Die Veröffentlichung von häufig frei zugänglichem Lehr- und Lernmaterial - insbesondere im Internet - hat in den letzten Jahren rapide zugenommen; diese online verfügbaren Materialien bezeichnen wir im vorliegenden Beitrag als ,alternative Unterrichtsmaterialien“. "Alternative" Unterrichtsmaterialien - eine begriffliche Annäherung: Unterrichtsmaterialien aus dem Internet lassen sich durch folgende grundsätzliche Eigenschaften charakterisieren (vgl. Matthes, 2014): Es sind didaktisch aufbereitete Darstellungen eines Themas, primär für die Zielgruppe der Lehrkräfte konzipiert und für den zeitlich begrenzten, partikularen Einsatz im Unterricht aufbereitet. Sie sind kostenlos ver- 
fügbar und häufig, wenn nicht von den Schulbuchverlagen zur Verfügung gestellt, keinem (staatlichen) Zulassungsverfahren unterworfen. Damit sind sie dem Ansatz der „Open Educational Resources“ (OER) sehr nah (zu Definitionsansätzen: Blees, Cohen \& Massar, 2013; Wiley, Bliss \& McEwen, 2014). Die Diskussion zur Qualität von OERs richtet sich dabei vor allem auf Fragen nach der Rolle des Nutzenden und auf Möglichkeiten der Qualitätssicherung (Brückner, 2018; Zawacki-Richter \& Mayrberger, 2017). Die Bezeichnung „alternativ" hebt einerseits auf die Verwendungsebene ab und meint alle Materialien, die Lehrkräfte potenziell nutzen können, da sie über die digitale Bezugsquelle frei zugänglich sind. Andererseits verweist er auf die Bandbreite des Ursprungs. Fey und Matthes (2015) konnten allein in den Jahren 2011 bis 2013 einen enormen Anstieg an Veröffentlichungen von digital verfügbaren Unterrichtsmaterialien beobachten und konstatieren eine $\mathrm{Zu}$ nahme von 854 auf 17.118 Angebote, die von Verlagen, Vereinen, Stiftungen, aber auch von Unternehmen zu unterschiedlichsten Themen zur Verfügung gestellt werden. Diese Angebote umfassen unterschiedlichste Formate und reichen von einzelnen Arbeitsblättern bis hin zu komplett ausgearbeiteten Unterrichtseinheiten inklusive didaktischen Umsetzungshinweisen und Handreichungen für Lehrkräfte.

Solche „alternativen“ Materialien haben das Potenzial, das Schulbuch als Medium zu ergänzen bzw. an manchen Stellen mit diesem sogar zu konkurrieren (vgl. Hiller, 2013). Von bildungspolitischer Seite wird dies als Chance postuliert: „Durch eine an die neu zur Verfügung stehenden Möglichkeiten angepasste Unterrichtsgestaltung werden die Individualisierungsmöglichkeit und die Übernahme von Eigenverantwortung bei den Lernprozessen gestärkt" (KMK, 2016, S. 12). Das Drängen anderer und teilweise neuer Akteure auf den Markt schulischer Materialien kann Vorteile bringen, wie die Aufbereitung ganz aktueller Themen oder spezifischer Akteursperspektiven, ist aber auch kritisch zu betrachten. Es kann vermutet werden, dass insbesondere Vereine, Stiftungen und Unternehmen spezifische lobbyistische Ziele verfolgen (Fey \& Matthes, 2015, S. 44; Verbraucherzentrale Bundesverband, 2014). Die von außerschulischen Akteuren angebotenen Unterrichtsmaterialien bilden „einen bestimmten Teil von gesellschaftlicher Realität" (ebd., S. 45) ab, geleitet durch die eigene Perspektive, die eigenen Interessen und die eigene Expertise. Problematisch wird dies, wenn (gerade in Unterrichtsmaterialien für jüngere Kinder) normative Positionierungen bspw. in Form von Verabsolutierungen von Standpunkten nicht transparent dargestellt werden (vgl. Fey, 2015, S. 214). Forderungen hinsichtlich einer zentralen Prüfung von alternativen Unterrichtsmaterialien wurden bisher nicht umgesetzt (vgl. Muuß-Merholz \& Schaumburg, 2014, S. 51). Auch für das Thema Flucht gibt es mittlerweile ein umfangreiches Angebot von Unterrichtsmaterialien, das vor allem von NROs in teilweise sehr unterschiedlicher Art und Weise gestaltet wird (vgl. Kater-Wettstädt, 2018).

"Alternative“ Unterrichtsmaterialien - im Unterricht: Der große Umfang des Angebots von digital zur Verfügung stehenden Unterrichtsmaterialien und die Bandbreite der unterschiedlichen Bezugsquellen birgt für Lehrkräfte die Herausforderung, zunächst auf in Frage kommende Materialien zu reduzieren und anschließend auf der Grundlage selbst entschiedener Qualitäts- kriterien auszuwählen. Das Angebot „alternativer“ Materialien - die i.d.R. nicht wie beispielsweise Schulbücher eine Approbation durchlaufen (vgl. Stöber, 2010) - rückt die Verantwortung und die Kompetenz des Nutzenden in den Fokus. In der entwicklungspolitischen Szene wurden Qualitätskriterien beschrieben (Überblick: Lange, 2017a, S. 123-124; Pädagogisches Werkstattgespräch, 2007; z.B. Stiftung Bildung und Entwicklung, 2012), die ein Angebot für Akteurinnen und Akteure in der Praxis zur Reflexion von Unterrichtsmaterialien darstellen können (vgl. Asbrand \& Lang-Wojtasik, 2009), aber nicht auf die Verwendung von Lehrenden zugeschnitten sind.

Eine adäquate Auswahl und Verwendung von „alternativen" Materialien birgt das Potenzial weitergehende Perspektiven und Expertise (vgl. Roncevic \& Hoffmann in diesem Heft) sowie innovative und kreative Zugänge zu Themen einzubinden. In der bildungspolitischen Einordnung wird hinsichtlich des Einbezugs der Materialien ein „Primat des Pädagogischen“ formuliert; damit ist gemeint, dass diese eingesetzt werden soll mit dem Ziel, curriculare Vorgaben einzulösen, eingesetzt werden sollen (KMK, 2016, S. 12). Die Ergebnisse der Studie von Neumann (2015) zeigen, dass eine Vielzahl von Lehrkräften angibt, dass sie, um ihren Unterricht interessant und zeitgemäß zu gestalten, häufig auf Materialien aus dem Internet zurückgreifen (Neumann, 2015; Verbraucherzentrale Bundesverband, 2014). Es wurden 720 Lehrkräfte aus unterschiedlichen Bereichen zu verwendeten Lehrmitteln befragt; dabei wird deutlich, dass Lehrkräfte Lehrmaterialien aus dem Internet vor allem nutzen, um sich „Anregungen für den eigenen Unterricht/eigenes Material“ (Neumann, 2015, S. 100) einzuholen. Über die Hälfte der befragten Lehrkräfte gibt als zweitwichtigste Funktion die "Arbeit mit Aufgaben“ (ebd.) an. Hier deutet sich an, dass alternative Materialien häufig der Ausgangspunkt für weitere Anpassungsschritte sind. Alternative digitale Materialien finden demnach graduell und in unterschiedlicher Gewichtung Eingang in den Unterricht. Die damit verbundene Herausforderung ist der Prozess der didaktischen Anpassung, Überarbeitung oder Weiterentwicklung z.B. vor dem Hintergrund der eigenen Lerngruppen (z.B. Peacock \& Gates, 2000).

Den Fragen danach, welche Rolle alternative Online-Materialien im Unterricht einnehmen können, wie Lehrkräfte mit den Materialien umgehen - also auch konkrete Strategien der Evaluation - werden vor dem Hintergrund des rasant angestiegenen digitalen Angebots für Lehrkräfte bisher wenig Aufmerksamkeit geschenkt. Die Nutzung wird derzeit ansatzweise im internationalen Lehrkräftebildungskontext (z.B. Clements \& Pawlowski, 2012; Sawyer \& Myers, 2018) bzw. im Hochschulkontext allgemein beforscht (z.B. Nikoi \& Armellini, 2012; Winitzky-Stephens \& Pickavance, 2017). Sawyer und Myers (2018) zeigen z.B. dass zunehmende Praxiserfahrungen von angehenden Lehrkräften den Gebrauch von Internetmaterial reduzieren. Clements und Pawlowski (2012) stellten in der Frage nach der Qualität von Internetmaterial fest, dass Lehrkräfte vor allem die Passung zum Curriculum als zentral beurteilten. Allgemeine Qualitätsstandards waren begrenzt bekannt und eigene Standards bezogen sich z.B. auf die Einbindung unterschiedlicher medialer Angebote oder auf die wissenschaftliche Korrektheit des Materials (vgl. ebd., S. 10f.). Strategien des Umgangs selbst wurden jedoch nicht herausgearbeitet. Ausgehend von diesem Desiderat steht im Zentrum 
dieses Beitrages die Frage, welche Auswahl- und Bewertungsstrategien (angehende) Sachunterrichtslehrkräfte mit einem im Internet zur Verfügung stehenden Unterrichtsmaterial zum Thema Flucht und Geflüchtete zeigen.

\section{Das Thema Flucht im (Sach-)Unterricht der Grundschule}

Flucht als komplexes gesellschaftliches Phänomen: Der Themenbereich Flucht und Geflüchtete kann exemplarisch für ein gesellschaftlich hoch aktuelles und politisches Thema gesehen werden, das zudem für die Auseinandersetzung mit gesellschaftlichen Entwicklungen im Sinne sozialer Nachhaltigkeit von zentraler Bedeutung ist. Trotz der langen deutschen Migrationsgeschichte wird Einwanderung, „(...) erst seit der Jahrtausendwende [...] als Faktum politischer Gestaltung (...)" postuliert (Meinhardt \& Schulz-Kaempf, 2015, S. 54). Dahinter verbirgt sich die zunehmende Gewissheit, dass eine Reduktion von Migration auf ein Phänomen der Wanderung unrealistisch ist, da Migration als „(...) mit der Veränderung und der Bestätigung des Bestehenden (...)“ (Castro Varela \& Mecheril, 2010, S. 35) einhergeht. Diese Veränderungen betreffen nicht nur die Gesellschaft als Ganzes, sondern stellen auch die bisherige Organisation von Schule und Unterricht, in Frage. Diese Frage jedoch, ob es „(...) um Erhalt oder Umgestaltung (...) (ebd.) von bestehenden Strukturen und Orientierungen aller beteiligten Akteure geht, wird kontrovers diskutiert. Des Weiteren ist Fluchtmigration ein vielschichtiges Thema, welches in der Diskussion um Ursachen gesellschaftliche, geographische, politische oder auch klimatische Aspekte umfasst (vgl. Oltmer, 2013).

Aktualität im Kontext Grundschule: Migrationsbewegungen sind historisch und auch weltweit eine Selbstverständlichkeit. Dies wird strukturell-organisatorisch und auch hinsichtlich curricular-inhaltlichen Fragen für Grundschulen relevant: zum einen durch zunehmend heterogene Lerngruppen und zum anderen im Hinblick auf den Bildungs- und Erziehungsauftrag der Grundschule. Laut dem Statistischen Bundesamt (2019) haben von den insgesamt 3,5 Mio. Kindern im Alter zwischen 5 bis 10 Jahren 1,4 Mio. einen Migrationshintergrund (ebd., S. 39). Das entspricht 40 Prozent der Kinder im Grundschulalter. Um spezifischer den Blick auf Kinder mit Fluchterfahrung zu richten, bietet der Umfang der für Grundschulkinder gestellten Asylanträge eine Orientierung. Maurice et al (2020) fassen die Daten des Bundesministeriums für Migration und Flüchtlinge zusammen: „In den Jahren 2015 bis 2017 wurden insgesamt 321.707 Asylanträge für Kinder unter 11 Jahren in Deutschland gestellt." Mit Blick auf den Familiennachzug ist anzunehmen, dass diese Zahlen in den letzten Jahren angestiegen sind.

Mit diesen Entwicklungen gehen, gerade auch im Hinblick auf Kinder mit Fluchthintergrund, Fragen hinsichtlich der Unterrichtung nicht alphabetisierter Kinder, Kinder ohne vorschulischer Bildung oder auch traumatisierter Kinder einher (Ritter \& Albers, 2016). Vor dem Hintergrund unserer Migrationsgesellschaft sind dabei Fluchterfahrungen und -migration nicht nur Kontext für pädagogisches Handeln, sondern auch zentrale Unterrichtsinhalte und sollten dementsprechend auch fester inhaltlicher Bestandteil der Ausbildung von Lehr- kräften sein (Lange, 2017b). Der Umgang mit Diversität, interkultureller Bildung oder Globalem Lernen fanden und finden zunehmend Eingang in die Kerncurricula als übergeordnete Aufgaben der Grundschule. Dabei nehmen gerade die Sprachbildung (Becker-Mrotzek \& Roth, 2017) und der Umgang mit Mehrsprachigkeit im Unterricht (Lange \& Pohlmann-Rother, 2020) eine zentrale Rolle ein.

Verortung des Themas Flucht im Sachunterricht: Sachunterrichtslehrkräfte stehen in der besonderen Verantwortung, Schülerinnen und Schüler dabei zu unterstützen, die Welt in Form vieler unterschiedlicher Themen mit den diversen sachunterrichtlichen Perspektiven zu erschließen (Kaiser, 2006; Stoltenberg, Holzmann \& Lienau, 2013). Sie unterliegen damit der Herausforderung der interdisziplinären und vielperspektivischen Aufbereitung von Unterrichtsinhalten, was die Notwendigkeit auf unterschiedliche Materialien zurückzugreifen gegebenenfalls noch dringlicher macht.

Schon Klafki (1996) forderte mit seinem Ansatz der Auseinandersetzung mit epochaltypischen Schlüsselproblemen die Thematisierung von Krieg und Frieden, womit die Themen Flucht und der Umgang mit Vielfalt als Resultate gesellschaftlicher Verschiebungsprozesse durch kriegerische Auseinandersetzungen eng verwoben sind (vgl. Kiel, 2018). In der curricularen Umsetzung des Sachunterrichts lassen sich entsprechende Anknüpfungspunkte finden. Inhalte der Thematik Fluchtmigration sind im Perspektivrahmen der GDSU (2013) beispielsweise im perspektivvernetzenden Themenbereich Mobilität („Unterscheidung zwischen verschiedenen Zwängen und Bedürfnissen der Mobilität"), in der sozialwissenschaftlichen Perspektive („Wahrnehmung der gesellschaftlichen Vielfalt") (Gläser \& Peuke, 2015), in der geographischen Perspektive („Vielfalt und Verflechtung von Räumen - Lebenssituationen nah und fern“) sowie in der historischen Perspektive („Alterität und Identität“) zu finden (GDSU, 2013).

Fluchtmigration in Schulbüchern und Unterrichtsmaterialien: Curricular sind die Themen Migration und Integration feste Bestandteile im Themenkanon von Schulbüchern und Unterrichtsmaterialien (z.B. Beauftragte der Bundesregierung für Migration, Flüchtlinge und Integration, 2015; Geuenich, 2015; Höhne, Kunz, Radtke \& Kugler, 2005). Häufig können Schulbücher für eine zeitgemäße Umsetzung von Themen, die rasanter gesellschaftlicher Veränderungen unterliegen - wie auch das Thema Flucht - aufgrund administrativer Prozesse jedoch nur begrenzt oder auch nur zeitversetzt Orientierung liefern.

Gomolla und Radtke (2002) stellten bereits vor 20 Jahren fest, dass Schulbücher und die ergänzenden Materialien sowohl Schülerinnen und Schülern als auch Lehrkräften „(...) mit Deutungsmustern aus dem Diskurs über Ausländer/ Migration/ Fremde/ Kulturkonflikt" (ebd., S. 274) versorgten. Wie die aktuellere Schulbuchstudie der Beauftragten der Bundesregierung für Migration, Flüchtlinge und Integration (2015) zeigt, thematisieren in Schulbüchern vorzufindende Darstellungen Einwanderung häufig (immer noch) vor allem problembehaftet und mit stereotypisierenden Betrachtungen zum Thema Flucht und Geflüchtete. Ähnliche Kritik üben aktuelle Studien im Hinblick auf rassistische Darstellungen von z. B. Subsahara Afrika in Schulbüchern (Awet, 2018). 
Dem steht eine lange Tradition in der Bildungsarbeit von Nichtregierungsorganisationen gegenüber, die sich dem interkulturellen oder globalen Lernen, der Bildung für nachhaltige Entwicklung, der Friedenserziehung, der Integrationsarbeit oder der Entwicklungspädagogik verschrieben haben. Entsprechend lassen sich viele Angebote von Lehrmaterialien zum Thema Flucht, wie oben beschrieben, finden (vgl. Kater-Wettstädt, 2018). Dabei zeigte sich, dass es ein besonderes Anliegen der NROs ist, gewissermaßen stellvertretend die Perspektiven von Geflüchteten oder die Sicht auf Geflüchtete anzubieten, z.B. durch authentische Selbstberichte oder abstrakte Schilderungen. Auch das Wissen um Aktionen und Handlungsmöglichkeiten steht im Fokus vieler dieser Materialien und reicht vom Aufzeigen lokaler Handlungsspielräume hin zur Unterstützung der Arbeit der herausgebenden NROs (ebd., S. 145ff.). Die Analyse der Materialien zeigt, dass die untersuchten Materialien überwiegend alternative Narrative zu der Thematisierung von Flucht und Migration im Unterricht bieten. Darin zeigen sich zum einen die Potenziale dieser Materialien wie die Darstellung verschiedener Perspektiven - sowie zum anderen Herausforderungen, wie eine mögliche Vereinnahmung für lediglich eine Narrative in der pädagogischen Aufarbeitung eines Themas.

\section{Methodisches Vorgehen}

Datenerhebung: Um der Frage nach den Evaluationsstrategien der (angehenden) Sachunterrichtslehrkräfte bezogen auf ein aus dem Internet zur Verfügung stehendes Unterrichtsmaterial zum Thema Flucht und Geflüchtete zu untersuchen, wurden zwischen 2016 und 2018 insgesamt 22 Gruppendiskussionen mit Studierenden des Faches Sachunterricht an der Universität Lüneburg sowie an der Universität Bamberg durchgeführt. Die Gruppendiskussionen wurden im Rahmen von sechs verschiedenen Seminaren erhoben und dauerten jeweils etwa 45-65 Minuten. Die Diskussionen fanden in Form von partizipativen „peer-Diskussionen“ statt; Dies bedeutet, dass die Studierenden die Diskussion nach einer kurzen Einführung mit vorgegebenen Frageimpulsen selbst leiteten. Der Gegenstand dieses Beitrags sind die Diskussionen zu der Frage: Was denken Sie über das Material? Vorab hatten die Studierenden den Auftrag sich mit dem online verfügbaren Unterrichtsmaterial zum Thema „Flucht" vertraut zu machen und auseinanderzusetzen.

Das eingesetzte Unterrichtsmaterial ${ }^{1}$, das für die Gruppendiskussionen genutzt wurde, besteht aus einem Schülerheft (insg. 26 Seiten) sowie einer zusätzlichen Handreichung für Lehrkräfte (vier Seiten), die beide frei online zugänglich und herunterladbar sowie in analoger Form verfügbar sind. Das Material ist explizit für die unterrichtliche Verwendung vorgesehen und - anders als die Vielzahl der Materialien - von staatlicher Seite geprüft. Das Material ist für die Sekundarstufe eins vorgesehen, jedoch können die Inhalte für den Einsatz in der Grundschule adaptiert werden. Diese Ausgangssituation sollte die Auseinandersetzung der Studierenden mit Fragen zur möglichen didaktischen Passung, zum Einsatz und zur Bewertung anregen. Das Material lässt sich in fünf Teile untergliedern: es beginnt mit einem „Wissenstest“ zum Thema „Was weißt du über Flüchtlinge?“, daraufhin folgen zwei Darstellungen von Prominenten und ihren Meinungen zum Thema Flucht, im
Anschluss findet man einen „Faktencheck“, der häufige Vorurteile aufgreift (z.B. „Unsere Gesellschaft kann so viele Flüchtlinge nicht verkraften") und Antworten darauf anbietet. Anschließend werden zwei Personen und ihr Fluchtweg sowie ihre Erfahrungen auf der Flucht beschrieben (gezeichnete Figuren, daher unklar, ob es sich um reale Gegebenheiten handelt); abschließend findet sich eine Linksammlung zu unterschiedlichen themenbezogenen Internetseiten.

Datenauswertung: Die Gruppendiskussionen wurden qualitativ-rekonstruktiv mit der Dokumentarischen Methode (Bohnsack, 2007) ausgewertet. Ziel der Auswertung ist die Annäherung an das Handlungswissen, also die impliziten Wissensbestände, die den Umgang mit einem Unterrichtsmaterial zu diesem Themenschwerpunkt bestimmen. Für die Dokumentarische Methode ist die an Karl Mannheims Wissenssoziologie (1980) angelehnte analytische Unterscheidung zwischen konjunktivem und kommunikativem Wissen zentral. Das kommunikative Wissen bezeichnet explizites, den Erforschten reflexiv verfügbares, theoretisches Wissen. Das konjunktive Wissen wird demgegenüber als implizites, atheoretisches Wissen verstanden. Die dokumentarische Methode bildet diese Unterscheidung in der Analyse durch zwei Interpretationsschritte ab (vgl. zum Folgenden ausführlich Bohnsack, 2007, S. 134 ff.). In der formulierenden Interpretation wird das kommunikative Wissen in der Betrachtung dessen, was gesagt oder getan wird, fokussiert und das sogenannte Orientierungsschema erfasst. In der reflektierenden Interpretation wird die Art und Weise, wie die Themen innerhalb der Gruppe diskutiert werden, in den Blick genommen. Durch die Beschreibung dieses „Wie“ der Diskussion lassen sich Rückschlüsse auf das konjunktive Wissen innerhalb der Gruppe ziehen, also auf ihren sogenannten Orientierungsrahmen. Diese analytische Trennung von theoretischem und atheoretischem Wissen, also zwischen Orientierungsschema und -rahmen wird in der Beschreibung von Orientierungsmustern wieder zusammengeführt (im Folgenden als Orientierungen beschrieben). Auf dieser Ebene der Rekonstruktion erfolgt eine sinngenetische Typenbildung, die Gegenstand der vorliegenden Analysen ist. Für die Analyse wurden in den Gruppendiskussionen Sequenzen identifiziert, die eine hohe Relevanz für die Fragestellung sowie eine erhöhte interaktive Dichte aufwiesen und fallintern und übergreifend nach dem Prinzip von minimalen und maximalen Kontrasten vergleichend analysiert. Die empirisch basierte komparative Analyse, als Kernelement der Dokumentarischen Methode (vgl. Bohnsack, 2007, S. 141ff.), erlaubt die Abstraktion vom Einzelfall und die Rekonstruktion der Orientierungsmuster über die Gruppen hinweg. Die in der rekonstruktiven Auswertung herausgearbeiteten Orientierungen werden folgend als Strategien der Studierenden zur Auswahl und Bewertung bezeichnet.

\section{Ergebnisse: Auswahl- und Bewer- tungsstrategien der Studierenden}

Im Rahmen der Gruppendiskussionen der Studierenden konnten zwei unterschiedliche Strategien zur Auswahl und Bewertung des Materials identifiziert werden: eine Orientierung an Praktikabilität und damit an einer direkten Verwertbarkeit des Materials und zum anderen eine Orientierung an Werten und 
übergeordneten Bildungszielen. Als übergeordneter kriterialer Fokus lässt sich in allen Gruppen die didaktische Frage der Passung zur Lerngruppe ausmachen, wobei häufig die Sinnhaftigkeit erwogen wird, für den Fall, dass Kinder mit Fluchterfahrungen in der Klasse wären. Die jeweilige Betrachtung der Passung des Materials für die Lerngruppe selbst unterliegt zwei grundlegenden Orientierungen. So werden von den Studierenden zwar ähnliche oder teils gleiche Kriterien an das Material angelegt, aber von einem jeweils anderen Ausgangspunkt bzw. übergeordnetem Interesse und Gestaltungsverständnis von unterrichtlicher Interaktion zu diesem Themenbereich: die einen Gruppen nehmen die Gestaltung einer adäquaten Interaktion zwischen den Lernenden und ihnen als Lehrkräfte als Ausgangpunkt und betrachten die Beschäftigung mit dem Material im Sinne einer Informationsquelle für die Lernenden. Die anderen Gruppen stellen die Gestaltung einer Wertebildung der Schülerinnen und Schüler in den Vordergrund, vor allem mit dem Fokus auf einen Perspektivenwechsel, der als moralisch-ethische Aufgabe und als Voraussetzung für tiefergehendes Verstehen gedeutet wird. Im Folgenden werden beide Orientierungen ausführlicher beschrieben.

\section{Orientierung an Praktikabilität (direkte Verwertbarkeit)}

Mit dieser pragmatischen Orientierung stellen die Studierenden die Praktikabilität und die direkte Verwertbarkeit des Materials in den Fokus. Die Passung der Inhalte für die Zielgruppe steht in diesen Diskussionen vor allem in methodischer Dimension im Vordergrund - also im Hinblick auf die angebotenen Aufgabenformate und die Durchführbarkeit der Vorschläge sowie der Aufgaben für die Lerngruppe. Die Gruppen explorieren und entwickeln in der Auseinandersetzung mit dem Material in ihren Augen angemessene Aufgabenformate, die stärker eine Passung zur Zielgruppe herstellen. In Abgrenzung zur zweiten Orientierung an übergeordneten Werten spielen in dieser ersten Orientierung Themen wie Perspektivenwechsel oder gegenseitiger Respekt zwar auch eine Rolle, werden jedoch als theoretische Schlagworte verwendet, die für die weitere Betrachtung des Materials und im Hinblick auf die Umsetzung selbst keine Rolle spielen. Im Folgenden werden anhand von beispielhaften Auszügen aus den unterschiedlichen Gruppendiskussionen die Reaktionen auf den Impuls nachgezeichnet.

Der erste Ausschnitt aus der Gruppendiskussion (Gruppe London $)^{2}$ zeigt die erste Positionierung zweier Studierender auf die Frage nach dem Material. Zuvor hatte eine Studierende kommentiert, dass die Begriffsklärungen sowie auch das Beispiel der Stellungnahme der prominenten Person positiv sind. Sie resümiert, dass das Material „als Info“ dienen könne. Dann schließt Dw mit ihrer Stellungnahme an.

\section{TK GD London, Z. 383-391}

Dw

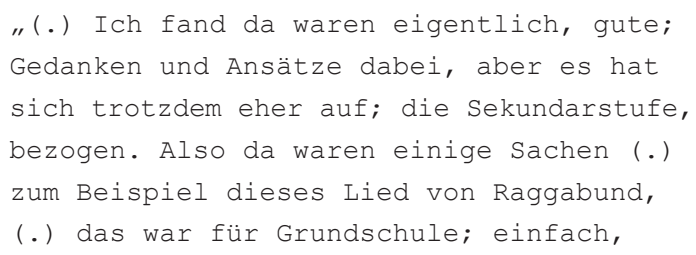

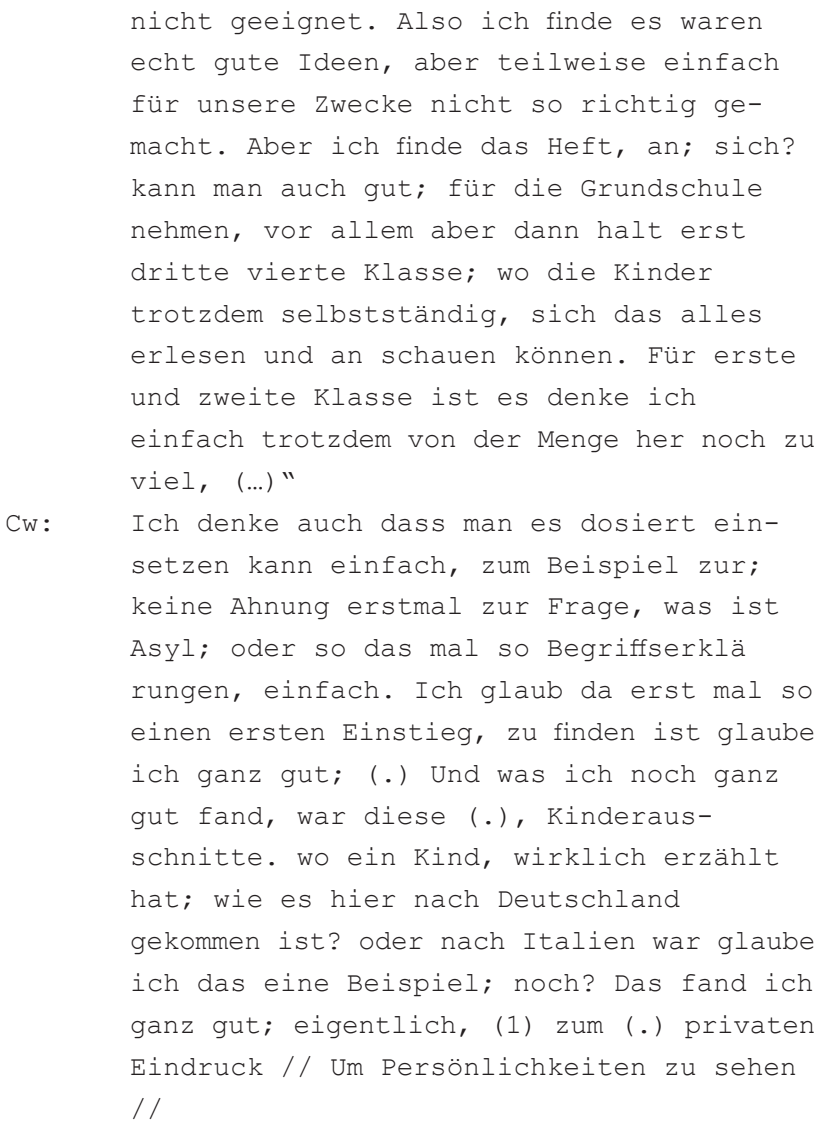

Dw elaboriert zunächst einmal den Bewertungsmodus, der durch die erste Studierende bereits gesetzt wurde und stellt fest, dass das Material inhaltlich gute Aspekte liefert, aber dass die Passung für die Grundschule nicht gegeben ist. Sie bezieht sich in ihrer Stellungnahme vor allem auf die Zielgruppenpassung, die auch anhand eines Beispiels („Lied von Raggabund“) negiert wird. Daran schließt sich ein Globalurteil über das Material an sich an, für welche Altersgruppe es geeignet erscheint. Sie löst damit einen pädagogischen Blick auf das Material ein, der das eigene professionelle Handeln bereits als auf die spezifische Zielgruppe der Grundschülerinnen und Grundschüler ausgerichtet markiert. Sie inkludiert dabei den Rest der Gruppe und markiert die adäquate Unterrichtung in der Grundschule als den gemeinsamen "Zweck“, gewissermaßen rahmt sie die Gruppe damit als eine Art „Zweckgemeinschaft“, wobei der Zweck in der adäquaten Beschäftigung der Kinder liegt. Die Kriterien, die der Zielgruppenadäquatheit zu Grunde gelegt werden, sind hier mehr oder weniger Formale: zum einen die Passung der Inhalte für die Kinder und damit verbunden der Umfang. Die Passung der Inhalte oder des Themas selbst oder was hier wichtige Lernziele wären, werden von Dw nicht in Frage gestellt; es ist eher eine Frage der „Menge“. Cw validiert die Aussage Dws bezüglich der partiellen Umsetzbarkeit des Materials. Die Begriffsklärungen werden hier als „Einstieg“ in das Thema ausdifferenziert. Damit würde sie die Integration des Materials zunächst auf die Entnahme der Definitionen beschränken. Hier wird der Horizont der grundsätzlichen Einsetzbarkeit des Materials ausgelotet, ausgehend von einem adäquaten strukturellen Aufbau als gelingende thematische Annäherung. Daran schließt $\mathrm{Cw}$ noch eine Bewertung eines Inhaltes (der „Kinderausschnitte“) an, was sie wiederum mit 
dem Zweck eines „privaten Eindrucks“ oder auch personalisierten Zugangs verknüpft. Auch in diesen Erwägungen geht es um die methodische Dimension zu der Frage, was für Kinder vermutlich anschlussfähig ist, nämlich die Verbindung mit (realen) Figuren („Persönlichkeiten“). Insgesamt zeigt sich damit bei $\mathrm{Cw}$ und $\mathrm{Dw}$ als übergeordnete Richtlinie die Zugänglichkeit für die Schülerinnen und Schüler in methodischer Hinsicht, das Material fungiert sozusagen als „Mittler“ der Informationen und Impulsgeber im Hinblick auf das Arrangement des Unterrichts, während sie sich selbst als Expertinnen für die Zielgruppe markieren. Um die konkreten Impulse des Materials herum wird ein „methodisches Gerüst“ gebaut, das die Umsetzung gewährleisten soll. Mit dem Herausgreifen einzelner Aufgabenformate wird ein eher eklektizistisches Vorgehen, also eine Entnahme von vereinzelten Teilen, wie der Begriffsdefinition und ein Rearrangieren und Anpassen für den möglichen Einsatz deutlich.

Der zweite Ausschnitt zeigt noch einmal eine andere Gruppe (Gruppe Vancouver), die sich auf gleiche Weise mit dem Material auseinandersetzt und einen konsensuellen Fokus auf die methodisch-funktionale Passung des Materials mit der Zielgruppe legt. Der Ausschnitt zeigt Dws erste ausführliche Annäherung an das Material, die in der Weiterführung einer methodischen Idee durch $\mathrm{Cw}$ aufgegriffen wird:

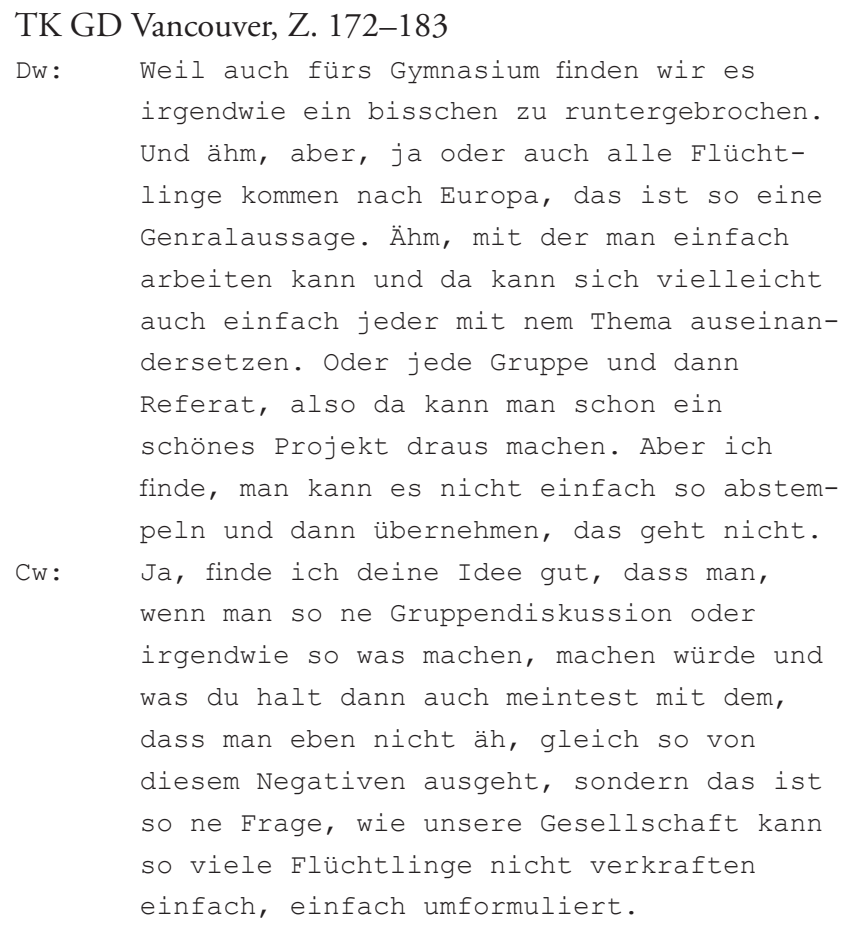

Die Aspekte der methodischen Passung zur Zielgruppe, die Dw und $\mathrm{Cw}$ hier in den Fokus rücken, sind denen aus dem Ausschnitt zuvor sehr ähnlich. Auch für Dw geht es zunächst darum, zu bestimmen für welche Zielgruppe das Material angemessen ist. Ausgehend von einer Aussage im Material, die zuvor bereits von ihr ausgeführt wurde, entwickelt sie umgehend eine Art Grobplanung von Unterricht, z.B. ein „schönes Projekt“ daraus zu machen und betont im Anschluss die Notwendigkeit der Adaption. Lernziele oder Erwartungen für die thematische Auseinandersetzung und Kompetenzen bleiben dabei außen vor. Die methodische Idee einer Gruppendiskussion wird über- nommen und soweit konkretisiert, dass ein entsprechender Impuls aber ebenfalls angepasst und „umformuliert“ werden muss. Bei beiden rückt damit der konkrete unterrichtliche Einsatz ins Zentrum, ein Explizieren oder Verhandeln von dem, was die Schülerinnen und Schüler tatsächlich lernen sollen, rückt wiederum fast gänzlich in den Hintergrund. Es geht der Gruppe vorrangig um die Gestaltung „schönen“ Unterrichts. Anders verhält sich das in den Gruppen, die ausgehend von einer Wertevermittlung über das Material diskutieren.

\section{Orientierung an bestimmten Werten und übergeordneten Bildungszielen}

Diese Orientierung ist an idealistischen Werten und übergeordneten Bildungszielen interessiert, die die Schülerinnen und Schüler in der Beschäftigung mit dem Thema nach Ansicht der Studierenden auf jeden Fall erlernen oder erreichen sollten. Die Studierenden diskutieren zwar auch die Passung zur Lerngruppe als ein wichtiges und zentrales Kriterium, aber die Passung wird hier ausgehend von einem übergeordneten Anliegen, von bestimmten Wertvorstellungen und Bildungszielen diskutiert, die im Rahmen der Auseinandersetzung mit dem Thema als zentral erachtet werden. Dabei rücken vor allem die Adäquatheit und Qualität der Inhalte - als dem Thema angemessen - in den Fokus, also die Frage, ob das Material der Problematik entsprechend gerecht wird. Dafür geht es nicht um die „Menge“ an Informationen, sondern um deren inhaltliche Beschaffenheit. Das zentrale Motiv, was sich hier wiederholt zeigt, sind die Bildungsziele „Empathie entwickeln“ und „Perspektivenwechsel“ einzuüben. Meist durchlaufen diese Gruppen den Perspektivwechsel sogar in der Gruppendiskussion selbst und schlüpfen gedanklich z. B. in die Rolle eines geflüchteten Kindes. Sie lösen damit selbst den Anspruch ein, den sie für eine angemessene Auseinandersetzung formulieren und bringen so gedankenexperimentell Motiv- oder Gefühlslagen Anderer in das Gespräch ein. In der Diskussion der Gruppe Teheran wird das an vielen Stellen deutlich. In der Auseinandersetzung findet sogar eine reflexive Abgrenzung zu einer reinen Wissensvermittlung statt; dies geschieht vor dem Hintergrund der ethischen Verantwortung, das Menschliche in diesem Zusammenhang in den Vordergrund zu stellen.

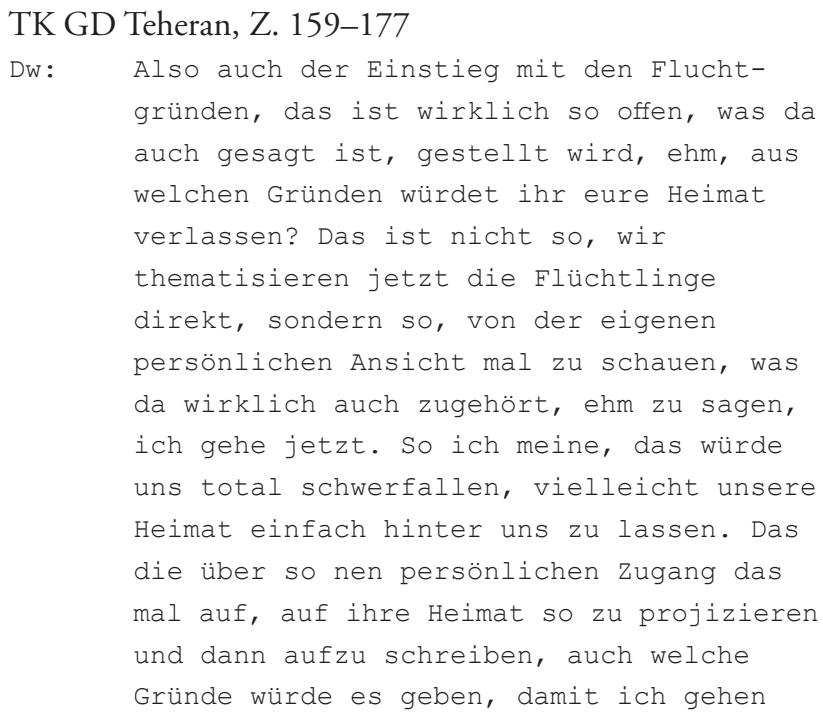




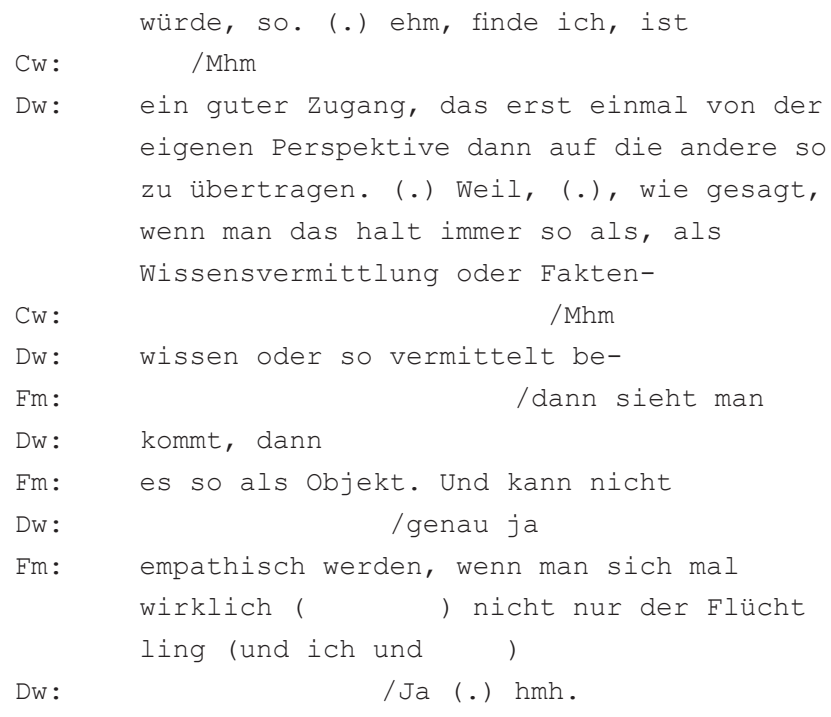

Ausgehend von der Aufgabenschilderung und dem im Material gestellten Impuls, stellt Dw den Kompetenzerwerb als Fähigkeit das eigene Verhalten in einer entsprechenden Situation zu reflektieren in den Vordergrund. Diese Fähigkeit wird wiederum als Voraussetzung für die Betrachtung der anderen Perspektive verstanden. Auch geht sie darauf ein, dass der Zugang deshalb sinnvoll ist, weil er nicht das Sprechen für eine Zielgruppe initiiert, sondern einen gemeinsamen Anlass anbietet, über beispielsweise das Thema Heimat zu sprechen. Gleich im Anschluss exploriert sie auch kurz diese (eigene) Perspektive, nämlich, dass es einem vermutlich sehr schwerfallen würde (zu fliehen). Die Erkundung der eigenen Emotionalität in dem Szenario „Gehen zu müssen" erlaubt dann eine in ihren Augen adäquate Übertragung auf die Perspektive von Menschen mit Fluchterfahrungen. Diesen Punkt der Reflexion - also die Art und Weise des Herangehens an das Thema - arbeitet sie gemeinsam mit Em aus. Sie beleuchten damit das Spannungsverhältnis zwischen Informationsvermittlung und Wertebildung im Hinblick auf das Verhältnis etwas als Objekt zu betrachten oder zum Subjekt zu machen. Dies macht deutlich, dass die höchste Priorität auf der Entwicklung von Empathie liegt, was Dw entsprechend validierend konkludiert. Um das wirkliche „Verstehen" der Situation von Menschen mit Fluchterfahrungen geht es auch in der folgenden Passage derselben Diskussion:

TK GD Teheran, Z. 216-224

$\mathrm{BW}$

/ Ich fand auch ganz gut, dass hier diese Geschichten nicht so (.) richtig, (.) äh richtig schlimm beschrieben werden, dass du jetzt fast anfängst zu heulen und so übelst Mitleid hast, sondern das, das ist da einfach beschrieben, wie ihr Weg war und das ist übelst krass, aber (.) es ist jetzt nicht so, dass man, also das die nur Mit-

\section{/ja/}

leid irgendwie ehm, jetzt erreichen wollen sondern das man das versteht, dass es echt heftig ist mal son, wenn man

/ja/

von zu Hause flüchten (.) muss
Die inhaltliche Qualität der geschilderten Erfahrungen bzw. der Geschichten, die in diesem Material aus der Sicht von zwei geflüchteten jungen Menschen dargestellt wird, beurteilt Bw anders als in der Gruppe Vancouver oder London im Hinblick auf das übergeordnete Ziel des Verstehens. Dabei geht es vordergründig darum, der Situation der Geflüchteten gerecht zu werden, und die Sachverhalte entsprechend adäquat abgebildet zu sehen, um ihre Perspektive übernehmen zu können. Mitleid wird dabei kritisch als wenig hilfreiche Perspektive auf die Zielgruppe eingeschätzt, die ggf. auch einem Verstehen im Wege stehen könnte. In der Gruppe wird hier also ein Impuls aus dem Material für die Reflexion des Bildungsauftrages im Sinne einer Wertevermittlung genutzt. Das Motiv der Perspektivenübernahme und des tatsächlichen Verstehens der Beweggründe der Flucht steht auch in dem folgenden Abschnitt aus der Diskussion „Accra“ im Zentrum. Hier wird zudem eine ethisch-moralische Verpflichtung direkt mit dem Thema verbunden.

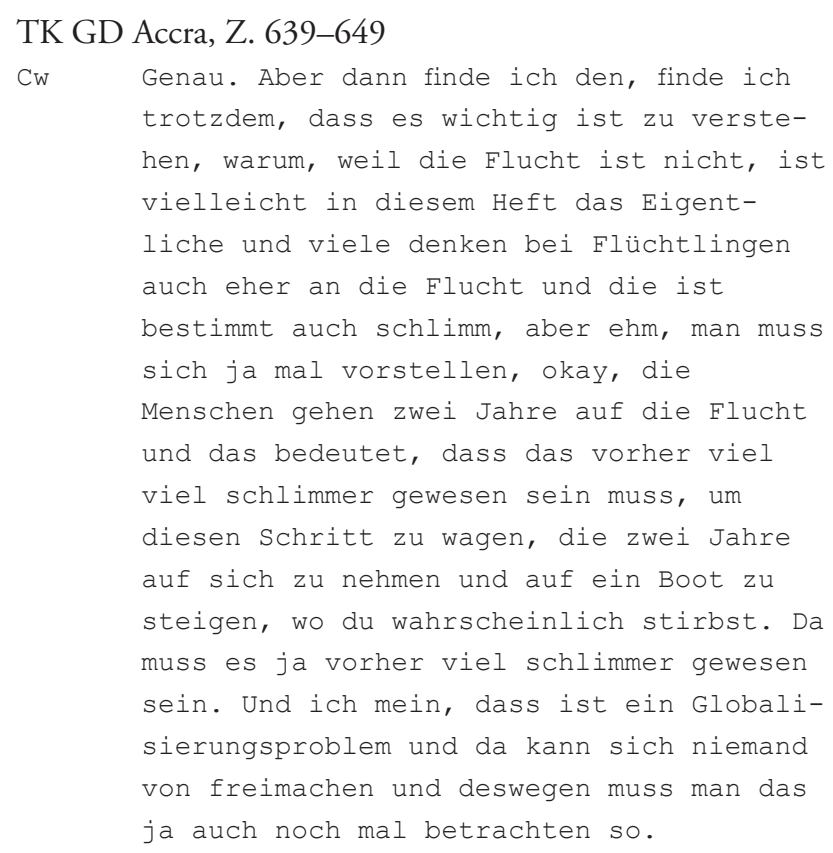

Cw aus der Gruppe Accra formuliert ihren Standpunkt hier recht spät im Diskussionsverlauf, nachdem bis dahin vor allem die kritische Reflexion und der Bezug eines eigenen Standpunktes aufgrund einer ausgewogenen Informationslage diskutiert wurden. Sie weitet dabei das Verstehen über das emotionale Nachempfinden hinaus auf den tiefergehenden Nachvollzug der Motivlage der geflüchteten Menschen, der im weiteren Verlauf von der Gruppe differenzierend elaboriert und konsensuell konkludiert wird. Sie macht deutlich, dass nicht nur die Fluchterfahrung selbst, sondern die Situation, die dazu geführt hat, noch schlimmer gewesen sein muss und negiert damit eine Betrachtung, die sich auf den Fluchtabschnitt reduziert. Dies tut auch sie mit einer Aufforderung die Perspektive zu wechseln (,ja mal vorstellen“) und sich in die Situation von Menschen, die sich auf die Flucht begeben, hineinzuversetzen. Sie vollzieht performativ selbst, was sie als Bildungsziel formuliert: die Perspektive zu ändern und Empathie zu entwickeln. Aus dieser Betrachtung heraus leitet sie eine globale Verantwortung ab, die die Thematisierung dieses „Problems“ legitimiert, unabhängig von der Lerngruppe: In 
ihren Augen geht dieses Thema jeden etwas an. In den dargestellten Abschnitten tritt damit die Lerngruppe relativ zurück und es geht vor allem um eine Adäquatheit der Darstellung, die der Problematik und dem übergeordneten Ziel, Empathie zu entwickeln und Perspektivübernahme einzuüben, gerecht werden muss.

Das Material und die darin enthaltenen Aufgaben werden hier vor allem für einen Austausch über zentrale Bildungsziele im Sinne einer Wertevermittlung, die mit diesem Thema verbunden sind, genutzt. Die Gruppe erarbeitet diese Perspektive z.B. noch in Bezug auf die Unangemessenheit des Titels („Was geht?") oder auch methodisch hinsichtlich des Einsatzes eines Rollenspiels und inhaltlich z.B. dahingehend wie der eigene Lebensstandard hinterfragt wird, um „(...)ja wirklich Perspektivenübernahme" zu schaffen (TK GD Accra, Z. 736).

\section{Rezeptiv-selektiver Umgang mit dem Material}

Unabhängig von diesen beiden unterschiedlichen, herausgearbeiteten Orientierungen zu den Auswahl- und Bewertungsstrategien der untersuchten Studierendengruppen ist hervorzuheben, dass die Gruppen insgesamt, eine rezeptiv-selektive Rolle gegenüber dem Material einnehmen. Dies zeigt sich in einer eher eklektizistischen und überwiegend unkritischen Herangehensweise an das Material.

Mögliche Alternativen wie etwa selbst das vorliegende Material umzugestalten oder das Material zu entwickeln, das den vorgebrachten Zielen besser entspricht, werden nicht angesprochen. Das Material eignet sich aus Sicht der Studierenden auch dazu, sich selbst Wissen zum Thema Flucht anzueignen, da viele die eigene Unwissenheit zum Thema als Herausforderung hervorheben. Der eklektizistische Umgang mit dem Material wird durch die Entnahme und Reflexion einzelner Aufgaben und Impulse deutlich, anhand derer Fragen zur methodischen Umsetzung oder zu Bildungszielen besprochen werden. Die einzelnen Teile, Aufgaben und Impulse werden als einzelne Angebote wahrgenommen und als solche auf ihre Nutzung und Ihre Passung für die Zielgruppe oder als inhaltlich adäquates Angebot für bestimmte angestrebte Kompetenzen diskutiert. Das Material wird damit vor allem in der Funktion des Anregens, Inspirierens und Vorschlagens bedeutsam, was einen partiellen Eingang in den Unterricht wahrscheinlich macht.

\section{Diskussion: Chancen und Herausforde- rungen in der Evaluation von Unterrichts- material zum Thema Flucht}

Im digitalen Zeitalter bietet das Internet Lehrkräften Zugang zu Unterrichtsmaterialien, in denen zeitgemäß und gerade auch politisch aktuelle Themen wie Fluchtmigration bearbeitet werden. Die Anforderungen an Lehrkräfte sind damit vielfältig in Bezug auf die Herausgeberschaften und damit ggf. bestimmten Realitätsausschnitten, also auf thematisch-inhaltlicher Ebene im Sinne der Beurteilung einer adäquaten Aufarbeitung und Abwägung der Darstellung und Bearbeitung des Themas Flucht sowie im Hinblick auf den potentiellen Einsatz im Unterricht.
Dieses Spannungsverhältnis zwischen methodisch-didaktischer und inhaltlicher Angemessenheit in der Beurteilung des Materials zeigt sich in den vorliegenden empirischen Ergebnissen. Während die einen vor allem die Umsetzung selbst und damit das Vorgehen, das in den Ausführungen des Materials nahegelegt wird, in den Blick nehmen, fokussieren die anderen Studierendengruppen vor allem die adäquate Darstellung des Themas, die sich aus dem Anspruch zum Perspektivenwechsel und damit verbunden der Entwicklung von Empathie ergibt. In dieser Fokussierung auf übergeordnete Bildungsziele zeigen sich zum einen Potenziale der Bearbeitung des Themas Flucht für eine multiperspektivische Auseinandersetzung mit globalen Fragestellungen, z.B. vor dem Hintergrund der Fluchtgründe. Zum anderen werden in den Ergebnissen Potenziale für eine Wertebildung sichtbar, die auf der Grundlage der Wahrnehmung und dem Nachvollzug anderer Sichtweisen ein selbstständiges und kritisches Urteilen und Position beziehen ermöglicht, wie es im Globalen Lernen, in der Bildung für nachhaltige Entwicklung (z.B. UNESCO, 2017; Wals \& Jickling, 2002), aber auch in der politischen Bildung (z.B. Forum kritische politische Bildung, 2019) und für den Sachunterricht im Allgemeinen gefordert wird. Das kritische Urteilen trägt bei zur „(...) Förderung der Autonomie des Individuums im Sinne der anspruchsvollen Ziele von Mit- und Selbstbestimmung sowie Solidarität" (Wulfmeyer, 2017a, S. 357). In der Orientierung an einer "direkten Verwertbarkeit“ von Unterrichtsmaterialien können sich auch Schwierigkeiten zeigen; nämlich hinsichtlich der möglichen inhaltlich unkritischen Übernahme von Inhalten, die weniger aus einem Bildungsanspruch heraus genutzt werden, als vielmehr als eine Art Hilfe für die adäquate Gestaltung der Interaktion fungieren und offener für die Übernahme der angebotenen Narrativen erscheinen. Eine Orientierung an übergeordneten Bildungszielen kann dabei ein Gegengewicht bilden zu einer allein pragmatischen Abwägung und einer stärker kompetenzorientierten Umsetzung.

Um für angehende Lehrkräfte hier eine Orientierung anzubieten, mag die Auseinandersetzung mit vorhandenen Qualitätssicherungsansätzen hilfreich sein, einerseits, weil sie auch Ansprüche der Bildungskonzepte konkretisieren (z.B. die unterschiedlichen Handlungsebenen, die globale Perspektive oder die Diversitätssensitivität, siehe z.B. SBE, 2012, auf übergeordneter Ebene z.B. Fey, 2015); andererseits, weil sie das reflexive Verhandeln und das Entwickeln eigener Evaluationsstrategien unterstützen kann. Auf der Grundlage von fundierten Qualitätskriterien und fachlichen Grundlagen zum Themenfeld Flucht und Migration können Studierende eine kritisch-reflexive Haltung entwickeln lernen. Dabei geht es darum, sowohl die eigene, subjektiv geprägte Perspektive als auch die Rolle anderer pädagogischer Akteure und ihrer Angebote zu reflektieren. Gerade die Intentionen von Herausgebenden gilt es dabei kritisch zu hinterfragen sowie die Passung des Angebots im Hinblick auf Kompetenzen, die Schülerinnen und Schüler erwerben sollen, zu überprüfen.

Eine offene Frage bleibt dabei wer zukünftig die aktive Ausgestaltung von Unterrichtsmaterialien bestimmt und was die „Open Access“-Entwicklungen für die Schule und Unterricht bedeuten. Für die in diesem Beitrag beschriebenen komplexen Aufgaben von Grundschullehrkräften in der Auswahl 
und Bewertung von „alternativen“ Unterrichtsmaterialien im digitalen Zeitalter erscheint die Bedeutung von Kooperation und Kollaboration besonders zentral. Multiprofessionelle $\mathrm{Zu}$ sammenschlüsse mit relevanten Akteuren aus u.a. Verlagen, Stiftungen, NROs - in die sich Lehrkräfte als aktive Gestaltende mit einbringen - können eine wertvolle Verbindung darstellen, um die Bildungsziele, die adäquate methodisch-didaktische Aufarbeitung und die verschiedenen disziplinären Perspektiven zusammen zu bringen, mit dem Ziel, qualitativ hochwertige Unterrichtsmaterialien zu entwickeln bzw. um Vorhandene zu bewerten.

\section{Anmerkungen}

1 Das Material ist verfügbar unter: https://www.bpb.de/shop/lernen/was-geht/221893 /was-geht-das-heft-zu-flucht-und-asyl (Stand: 8.7.2020). Gefördert durch die Qualitätsoffensive Lehrerbildung des BMBF, 1. Förderphase von 2016-2019.

2 Die Anonymisierung des Datenmaterials erfolgte über eine zufällige Zuordnung von Namen internationaler Großstädte. Die Sprecher wurden in jeder Gruppendiskussion mit A beginnend anonymisiert. Der Zusatz w (weiblich) oder m (männlich) gibt das Geschlecht der Person an. Die Lehrmittelreihe "Querblicke" basiert auf einem Verständnis von BNE als Vermittlung von spezifischen Kompetenzen nach Künzli and Bertschy (2008). Vgl hierzu auch https://www.querblicke.ch

\section{Literatur}

Asbrand, B. \& Lang-Wojtasik, G. (2009). Qualitätskriterien für Unterrichtsmaterialien entwicklungsbezogener Bildungsarbeit. Zeitschrift für internationale Bildungsforschung und Entwicklungspädagogik, 32(2), 8-13.

Awet, K. (2018). Die Darstellung Subsahara-Afrikas im deutschen Schulbuch. Gesellschaftslehre, Erdkunde, Geschichte und Politik der Sekundarstufe I (Gesamtschule) in Nordrhein-Westfalen. Opladen:BudrichUniPressLtd.https://doi.org/10.3224/86388 799

Beauftragte der Bundesregierung für Migration, Flüchtlinge und Integration. (2015). Schulbuchstudie Migration und Integration. Berlin. Aufruf am 17.07.2020 https://repository.gei.de/handle/11428/65

Becker-Mrotzek, M. \& Roth, H.-J. (Hrsg.) (2017). Sprachliche Bildung - Grundlagen und Handlungsfelder (Sprachliche Bildung, Band 1). Münster: Waxmann.

Blees, I., Cohen, N. \& Massar, T. (2013). Freie Bildungsmedien (OER). Dossier: Offene Bildungsressourcen / Open Educational Resources - Handlungsfelder, Akteure, Entwicklungsoptionen in internationaler Perspektive (Stand: Juni 2013). Frankfurt am Main: Deutsches Institut für Internationale Pädagogische Forschung.

Bohnsack, R. (2007). Rekonstruktive Sozialforschung. Einführung in qualitative Methoden (6., durchgesehene und aktualisierte Auflage). (UTB. 8242: Erziehungswissenschaft, Sozialwissenschaft). Opladen: Budrich.

Brückner, J. (2018). Eine Frage der Qualität. Qualitätsforderungen an Open Educational Resources in Schule und Hochschule. MedienPädagogik: Zeitschrift für Theorie undPraxisderMedienbildung, (32), 51-62.https://doi.org/10.21240/mpaed/32/201 8.10.23.X

Castro Varela, M. d. M. \& Mecheril, P. (2010). Grenze und Bewegung. Migrationswissenschaftliche Klärungen. In P. Mecheril, A. Kalpaka, C. Melter, İ. Dirim \& M. d. M. Castro Varela (Hrsg.), Migrationspädagogik (Studium Paedagogik, S. 23-53). Weinheim: Beltz Verlag.

Clements, K. I. \& Pawlowski, J. M. (2012). User-Oriented Quality for OER: Understanding Teachers' Views on Re-Use, Quality, and Trust. Journal of Computer Assisted Learning, 28(1), 4-14. https://doi.org/10.1111/j.1365-2729.2011.00450.x

Fey, C.-C. (2015). Kostenfreie Online-Lehrmittel. Eine kritische Qualitätsanalyse (Beiträge zur historischen und systematischen Schulbuch- und Bildungsmedienforschung). Bad Heilbrunn: Klinkhardt.

Fey, C.-C. \& Matthes, E. (2015). Unterrichtsmaterialien von Unternehmen und Vereinen. Zielsetzung und zentrale Ergebnisse der Augsburger Studie. Pädagogik, 67(10), 44-47.

Forum kritische politische Bildung. (2019). Bildung macht Zukunft. Positionen zu einer zukunftsorientierten kritisch-emanzipatorischen Bildung. Zugriff am 17.07.2020 https://akg-online.org/sites/default/files/bildung_macht_zuukunft_2019_-_positi onspapier.pdf

GDSU. (2013). Perspektivrahmen Sachunterricht (Vollständig überarbeitete und erweiterte Ausgabe). Bad Heilbrunn: Klinkhardt.

Geuenich, H. (2015). Migration und Migrant(inn)en im Schulbuch. Diskursanalysen nordrhein-westfälischer Politik- und Sozialkundebücher für die Sekundarstufe I (Inter- kulturelle Studien). Wiesbaden: Springer VS. https://doi.org/10.1007/978-3-658-0 6688-8

Gläser, E. \& Peuke, J. (2015). Migration und Migrationsgesellschaft im sozialwissenschaftlichen Sachunterricht thematisieren. In E. Gläser \& D. Richter (Hrsg.), Die sozialwissenschaftliche Perspektive konkret. Begleitband 1 zum Perspektivrahmen Sachunterricht (S. 151-169). Bad Heilbrunn: Verlag Julius Klinkhardt.

Gomolla, M. \& Radtke, F.-O. (2002). Institutionelle Diskriminierung. Die Herstellung ethnischer Differenz in der Schule. Wiesbaden: VS Verlag für Sozialwissenschaften. https://doi.org/10.1007/978-3-322-97400-6_2

Hiller, A. (2013). Der Einfluss des Internets auf das Steuerungspotenzial von Staat und Schulbuch in der Schulbildung. In E. Matthes, S. Schütze \& W. Wiater (Hrsg.), Digitale Bildungsmedien im Unterricht (S. 42-52). Bad Heilbrunn: Verlag Julius Klinkhardt.

Höhne, T., Kunz, T., Radtke, F.-O. \& Kugler, M. (Hrsg.). (2005). Bilder von Fremden. Was unsere Kinder aus Schulbüchern über Migranten lernen sollen (Frankfurter Beiträge zur Erziehungswissenschaft Reihe Monographien, Bd. 3). Frankfurt am Main: Johann-Wolfgang-Goethe-Universität.

Kaiser, A. (2006). Sachunterricht der Zukunft ist Weltorientierung. In S. Pfeiffer (Hrsg.), Neue Wege im Sachunterricht (Oldenburger Vor-Drucke. 551, S. 189-208). Oldenburg: Didaktisches Zentrum.

Kater-Wettstädt, L. (2018). Unterricht zum Thema „Flucht und Flüchtlinge“? Eine Analyse von Lehrmaterialien aus dem Internet. Zeitschrift für Bildungsforschung, 8(2), 137-152. https://doi.org/10.1007/s35834-018-0217-8

Kiel, E. (2018). Schlüsselprobleme weiter denken! In K.-H. Braun, F. Stübig \& H. Stübig (Hrsg.), Erziehungswissenschaftliche Reflexion und pädagogisch-politisches Engagement. Wolfgang Klafki weiterdenken (S. 109-125). Wiesbaden: Springer VS. https://doi.org/10.1007/978-3-658-18595-4_10

Klafki, W. (1996). Grundzüge eines neuen Allgemeinbildungskonzepts. Im Zentrum: Epochaltypische Schlüsselprobleme. In W. Klafki (Hrsg.), Neue Studien zur Bildungstheorie und Didaktik. Zeitgemäße Allgemeinbildung und kritisch-konstruktive Didaktik (S. 43-81). Weinheim: Beltz.

KMK. (2016). Bildung in der digitalen Welt. Strategie der Kultusministerkonferenz. Beschluss der Kultusministerkonferenz vom 08.12.2016 in der Fassung vom 07.12.2017. Berlin: Ständige Konferenz der Kultusminister der Länder in der Bundesrepublik Deutschland. Zugriff am 17.07.2020 https://www.kmk.org/fileadmin/Dateien/ pdf/PresseUndAktuelles/2017/Strategie_neu_2017_datum_1.pdf

Lange, S. (2017a). Globales Lernen - Bildungsmaterialien. In G. Lang-Wojtasik \& U. Klemm (Hrsg.), Handlexikon globales Lernen (2. überarbeitete und erweiterte Auflage, S. 122-126). Münster: Klemm \& Oelschläger.

Lange, S. (2017b). Professionalisierung der Pädagogischen Flüchtlingsarbeit. Empirische Einblicke zur Sicht von Studierenden aus, Service Learning-Seminaren. Zeitschrift für internationale Bildungsforschung und Entwicklungspädagogik, 40(1), 32-37.

Lange, S. \& Pohlmann-Rother, S. (2020). Überzeugungen von Grundschullehrkräften zum Umgang mit nicht-deutschen Erstsprachen im Unterricht. Zeitschrift für Bildungsforschung, 10(1), 43-60. https://doi.org/10.1007/s35834-020-00265-4

Mannheim, K. (1980). Strukturen des Denkens (Suhrkamp-Taschenbuch Wissenschaft, Bd. 298). Frankfurt am Main: Suhrkamp.

Matthes, E. (2014). Kostenlose Bildungsmedien online. Massenphänomen im NetzLösen sie das Schulbuch ab? Hamburg: Onlinedokument von der Universität Augsburg; Lehrstuhl für Pädagogik.

Maurice v., M, Balaban, E., Will, G. \& Roßbach, H.-G. (2020). Kinder mit Fluchthintergrund: Zur Bedeutung von Kindertageseinrichtungen für einen erfolgreichen Übergang in die Grundschule und den späteren Bildungserfolg. In Pohlmann-Rother, S., Lange, S. D. \& Franz, U. (Hrsg.). (2020). Kooperation von KiTa und Grundschule. Digitalisierung, Inklusion und Mehrsprachigkeit-Aktuelle Herausforderungen beim Übergang bewältigen (Bd. 2, S. 45-80). Köln: Carl Link.

Meinhardt, R. \& Schulz-Kaempf, W. (2015). Einwanderungen nach Deutschland und Migrationsdiskurse in der Bundesrepublik - eine Synopse. In R. Leiprecht \& A. Steinbach (Hrsg.), Grundlagen - Diversität-Fachdidaktiken (S. 54-92). Schwalbach: Debus Pädagogik.

Muuß-Merholz, J. \& Schaumburg, F. (2014). Open Educational Resources (OER) für Schulen in Deutschland 2014. Whitepaper zu Grundlagen, Akteuren und Entwicklungen. Berlin: Internet \& Gesellschaft Collaboratory e.V. Zugriff am 17.07.2020 http://www.collaboratory.de/images/6/64/OER-Whitepaper_2014einseitig.pdf; http://www.joeran.de/dox/2.-OER-Whitepaper_OER-f\%C3\%BCr-Schulen-inDeutschland-2014.pdf

Neumann, D. (2015). Bildungsmedien Online. Kostenloses Lehrmaterial aus dem Internet. Marktsichtung und empirische Nutzungsanalyse (Beiträge zur historischen und systematischen Schulbuch- und Bildungsmedienforschung). Bad Heilbrunn: Klinkhardt.

Nikoi, S. \& Armellini, A. (2012). The OER Mix in Higher Education: Purpose, Process, Product, and Policy. Distance Education, 33(2), 165-184. https://doi.org/ $10.1080 / 01587919.2012 .697439$ 
Oltmer, J. (2013). Migration im 19. und 20. Jahrhundert (Enzyklopädie Deutscher Geschichte, Band 86, 2. Auflage). München: Oldenbourg Verlag. https://doi.org/10 $1524 / 9783486756005$

Peacock, A. \& Gates, S. (2000). Newly Qualified Primary Teachers' Perceptions of the Role of Text Material in Teaching Science. Research in Science and Technological Education, 18(2), 155-171. https://doi.org/10.1080/713694982

PWG - Pädagogisches Werkstattgespräch. (2007). Beurteilungskriterien für Unterrichtsmaterialien des "Globalen Lernens". Jahrbuch Globales Lernen 2007/2008: Bielefeld: Welthaus Bielefeld.

Ritter, E. \& Albers, T. (2016). Kinder mit Fluchterfahrung in Kita und Grundschule. Zugriff am 7.8.2020 https://www.kita-fachtexte.de/fileadmin/Redaktion/Publikationen//KiTaFT_Ritter_Albers_Flucht_2016.pdf

Sawyer, A. G. \& Myers, J. (2018). Seeking Comfort: How and Why Preservice Teachers Use Internet Resources for Lesson Planning. Journal of Early Childhood Teacher Education, 39(1), 16-31. https://doi.org/10.1080/10901027.2017.138762

SBE - Stiftung Bildung und Entwicklung. (2012). Qualitätskriterien der SBE für die Evaluation von Unterrichtsmaterialien. Bern: education 21 - Bildung für Nachhaltige Entwicklung.

Statistisches Bundesamt. (2019). Bevölkerung und Erwerbstätigkeit. Bevölkerung mit Migrationshintergrund. Ergebnisse des Mikrozensus 2018. Wiesbaden: Statistisches Bundesamt (Destatis).

Stöber, G. (2010). Schulbuchzulassung in Deutschland. Grundlagen, Verfahrensweisen und Diskussionen. Braunschweig: Georg-Eckert-Inst. für internationale Schulbuchforschung. Zugriff am 17.07.2020 http://d-nb.info/1002260256/34; https:// nbn-resolving.org/urn:nbn:de:0220-2010-00146

Stoltenberg, U., Holzmann, V. \& Lienau, R. (2013). Bildung für eine nachhaltige Entwicklung in Schulen. Herausforderungen für die LehrerInnenbildung; eine Tagungsdokumentation als Beitrag zur Qualitätsentwicklung von Schulen und Unterricht und der Professionalisierung von Lehrerinnen und Lehrern unter dem Anspruch von Bildung für eine nachhaltige Entwicklung. Lüneburg: Univ.

UNESCO. (2017). Education for sustainable development goals. Learning objectives. Paris: UNESCO.

VZBV - Verbraucherzentrale Bundesverband. (2014). Unterrichtsmaterial unter der Lupe. Wie weit geht der Lobbyismus in Schulen? Eine Qualitätsanalyse von Lehrmaterialien verschiedener Anbieter und Interessenvertreter der Verbraucherzentrale Bundesverband (vzbv). Berlin: 0 . $V$.

Wals, A. E. J. \& Jickling, B. (2002). "Sustainability" in higher education: From doublethink and newspeak to critical thinking and meaningful learning. Internati- onal Journal of Sustainability in Higher Education, 3(3), 221-232. https://doi.org/10 $1108 / 14676370210434688$

Wiley, D., Bliss, T. J. \& McEwen, M. (2014). Open Educational Resources: A Review of the Literature. In J. M. Spector, M. D. Merrill, J. Elen \& M. J. Bishop (Hrsg.), Handbook of Research on Educational Communications and Technology (S. 781-789). New York, NY: Springer New York. https://doi.org/10.1007/978-14614-3185-5_63

Winitzky-Stephens, J. \& Pickavance, J. (2017). Open Educational Resources and Student Course Outcomes: A Multilevel Analysis. International Review of Research in Open and Distributed Learning, 18(4), 35-49. Zugriff am 17.07.2020 https:// eric.ed.gov/?id=EJ1146207 https://doi.org/10.19173/irrodl.v18i4.3118

Wulfmeyer, M. (2017). Sachunterricht der Grundschule und Globales Lernen. In G. Lang-Wojtasik \& U. Klemm (Hrsg.), Handlexikon globales Lernen (2. überarbeitete und erweiterte Auflage, 356-360). Münster: Klemm \& Oelschläger.

Zawacki-Richter, O. \& Mayrberger, K. (2017). Qualität von OER. Internationale Bestandsaufnahme von Instrumenten zur Qualitätssicherung von Open Educational Resources (OER) - Schritte zu einem deutschen Modell am Beispiel der Hamburg Open Online University (Sonderband zum Fachmagazin Synergie). Hamburg: Universität Hamburg Universitätskolleg.

\section{Dr. ${ }^{\text {in }}$ Lydia Kater-Wettstädt}

Verwaltungsprofessorin für „Trans- und Interkulturelle Bildung und Sachunterricht" am Institut für Integrative Studien der Leuphana Universität Lüneburg. Forschungsschwerpunkte: Migration und Flucht im Unterricht, Bildung für nachhaltige Entwicklung und Globales Lernen in der Lehrkräftebildung.

\section{Dr. ${ }^{\text {in }}$ Sarah Désirée Lange}

ist Akademische Rätin mit Forschungsausrichtung am Lehrstuhl für Grundschulpädagogik und Grundschuldidaktik an der Julius-Maximilians-Universität Würzburg. Ihre Forschungsschwerpunkte sind Mehrsprachigkeitsforschung, Fluchtmigration in der Grundschule, Unterrichtsqualität und Professionalisierung von Lehrkräften, International und vergleichende Forschung. In ihrem aktuellen Forschungsprojekt BLUME untersucht sie die Überzeugungen von Grundschullehrkräften zum Umgang mit Mehrsprachigkeit im Unterricht.

\section{UNSERE BUCHEMPFEHLUNG}

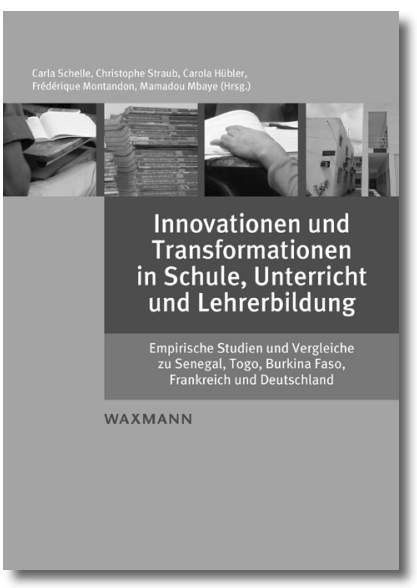

2020, 238 Seiten, br., $34,90 €$ ISBN 978-3-8309-4137-8

E-Book: 30,99

ISBN 978-3-8309-9137-3
Carla Schelle, Christophe Straub, Carola Hübler, Frédérique Montandon, Mamadou Mbaye (Hrsg.)

\section{Innovationen und Transformationen in Schule, Unterricht und Lehrerbildung \\ Empirische Studien und Vergleiche zu Senegal, Togo, \\ Burkina Faso, Frankreich und Deutschland}

Dieser Band versammelt Beiträge zu methodischen, didaktischen und pädagogischen Innovationen in den Praxisfeldern Schule, Unterricht und Lehrerbildung in Frankreich, Deutschland, Senegal, Togo und Burkina Faso. In den überwiegend empirischen, teils vergleichenden Beiträgen werden Traditionslinien, transformative Prozesse, komplexe Implikationen sowie Chancen und Hürden bei der Umsetzung innovativer Projekte in unterschiedlichen Länderbeispielen deutlich. Es werden damit auch Einblicke in kulturelle Kontexte ermöglicht und Befunde sichtbar, die im deutschsprachigen Raum nicht ohne Weiteres zugänglich sind. 